\title{
Coefficient estimates and Fekete-Szegö inequality for a class of analytic functions satisfying subordinate condition associated with Chebyshev polynomials
}

Eszter Szatmari

Department of Mathematics, Faculty of Mathematics and Computer

Science, Babeş-Bolyai University, Cluj-Napoca, Romania

email: szatmari.eszter@math.ubbcluj.ro

\author{
Şahsene Altinkaya \\ Department of Mathematics, \\ Faculty of Arts and Science, \\ Bursa Uludag University, \\ Bursa, Turkey
}

email: sahsene@uludag.edu.tr

Abstract. In this paper, we define a class of analytic functions, $\mathcal{F}(\mathcal{H}, \alpha, \delta, \mu)$, satisfying the following condition

$$
\left(\alpha\left[\frac{z f^{\prime}(z)}{f(z)}\right]^{\delta}+(1-\alpha)\left[\frac{z f^{\prime}(z)}{f(z)}\right]^{\mu}\left[1+\frac{z f^{\prime \prime}(z)}{f^{\prime}(z)}\right]^{1-\mu}\right) \prec \mathcal{H}(z, t),
$$

where $\alpha \in[0,1], \delta \in[1,2]$ and $\mu \in[0,1]$.

We give coefficient estimates and Fekete-Szegö inequality for this class.

\section{Introduction}

Let $\mathcal{A}$ denote the class of analytic functions in the open unit disk $\mathbb{U}=\{z:|z|<$ $1\}$, of the form

$$
f(z)=z+\sum_{k=2}^{\infty} a_{k} z^{k}, z \in \mathbb{U} .
$$

2010 Mathematics Subject Classification: 30C45, 30C50, 30C80

Key words and phrases: analytic functions, subordination, Chebyshev polynomials, coefficient estimates, Fekete-Szegö inequality 
Definition 1 [7, p.4] Let $\mathrm{f}, \mathrm{g}$ analytic functions in the open unit disk. The function $\mathrm{f}$ is said to be subordinate to $\mathrm{g}$, written $\mathrm{f} \prec \mathrm{g}$, or $\mathrm{f}(z) \prec \mathrm{g}(z)$, if there exists a function $w$, analytic in $\mathbb{U}$, with $w(0)=0$ and $|w(z)|<1$ (i.e. $w$ is a Schwarz function), such that $\mathrm{f}(z)=\mathrm{g}[w(z)], z \in \mathbb{U}$.

Remark 1 [7, p.4] If $\mathrm{g}$ is univalent, then $\mathrm{f} \prec \mathrm{g}$ if and only if $\mathrm{f}(0)=\mathrm{g}(0)$ and $\mathrm{f}(\mathbb{U}) \subset \mathrm{g}(\mathbb{U})$.

Chebyshev polynomials are of four kinds, but the most common are the Chebyshev polynomials of the first kind,

$$
\mathrm{T}_{\mathrm{n}}(x)=\cos n \theta, x \in[-1,1],
$$

and the second kind,

$$
u_{n}(x)=\frac{\sin (n+1) \theta}{\sin \theta}, x \in[-1,1],
$$

where $\mathrm{n}$ denotes the polynomial degree and $x=\cos \theta$.

Applications of Chebyshev polynomials for analytic functions can be found in $[1,2,3,4]$.

Let

$$
\mathcal{H}(z, \mathrm{t})=\frac{1}{1-2 \mathrm{t} z+z^{2}},
$$

where $t=\cos \theta, \theta \in\left(-\frac{\pi}{3}, \frac{\pi}{3}\right)$.

We have

$$
\begin{aligned}
\mathcal{H}(z, \mathrm{t}) & =1+\sum_{n=1}^{\infty} \frac{\sin (\mathrm{n}+1) \theta}{\sin \theta} z^{\mathrm{n}} \\
& =1+2 \cos \theta z+\left(3 \cos ^{2} \theta-\sin ^{2} \theta\right) z^{2}+\cdots \\
& =1+\mathrm{U}_{1}(\mathrm{t}) z+\mathrm{U}_{2}(\mathrm{t}) z^{2}+\cdots, z \in \mathbb{U}, \mathrm{t} \in\left(\frac{1}{2}, 1\right]
\end{aligned}
$$

where

$$
\mathrm{u}_{\mathrm{n}-1}=\frac{\sin \left(\mathrm{n} \cos ^{-1} \mathrm{t}\right)}{\sqrt{1-\mathrm{t}^{2}}}, \mathrm{n} \in \mathbb{N},
$$

are the Chebyshev polynomials of second kind.

Furthermore, we know that

$$
u_{n}(t)=2 t U_{n-1}(t)-U_{n-2}(t)
$$


and

$$
\mathrm{U}_{1}(\mathrm{t})=2 \mathrm{t}, \mathrm{U}_{2}(\mathrm{t})=4 \mathrm{t}^{2}-1, \cdots
$$

In this paper, we define a new class of analytic functions, being motivated by the following result.

Corollary 1 [5] Let $\mathrm{f} \in \mathcal{A}$ and also let $\alpha \in[0,1], \mathrm{a} \in[0,1], \delta \in[1,2]$ and $\mu \in[0,1]$. If

$$
\mathfrak{R}\left(\alpha\left[\frac{z f^{\prime}(z)}{f(z)}\right]^{\delta}+(1-\alpha)\left[\frac{z f^{\prime}(z)}{f(z)}\right]^{\mu}\left[1+\frac{z f^{\prime \prime}(z)}{f^{\prime}(z)}\right]^{1-\mu}\right)>a, z \in \mathbb{U},
$$

then

$$
\mathfrak{R}\left(\frac{z f^{\prime}(z)}{f(z)}\right)>a, z \in \mathbb{U},
$$

so $f$ is starlike of order a in $\mathbb{U}$.

Definition 2 We say that $\mathrm{f} \in \mathcal{A}$ of the form (1) belongs to $\mathcal{F}(\mathcal{H}, \alpha, \delta, \mu)$ if

$$
\left(\alpha\left[\frac{z f^{\prime}(z)}{f(z)}\right]^{\delta}+(1-\alpha)\left[\frac{z f^{\prime}(z)}{f(z)}\right]^{\mu}\left[1+\frac{z f^{\prime \prime}(z)}{f^{\prime}(z)}\right]^{1-\mu}\right) \prec \mathcal{H}(z, t),
$$

the power is considered to have principal value, $\alpha \in[0,1], \delta \in[1,2]$ and $\mu \in$ $[0,1]$.

Taking $\alpha=\delta=\mathrm{t}=1$ and $w(z)=z$, we obtain the following example.

Example 1 The function $f(z)=\frac{z}{1-z} e^{\frac{z}{1-z}}$ with the series expansion $f(z)=$ $z+2 z^{2}+\frac{7}{2} z^{3}+\cdots$ belongs to $\mathcal{F}(\mathcal{H}, \alpha, \delta, \mu)$.

For the purpose of our results, we need the following lemma.

Lemma 1 [6] Let the Schwarz function $w$ be given by

$$
w(z)=w_{1} z+w_{2} z^{2}+w_{3} z^{3}+\cdots, z \in \mathbb{U} .
$$

Then

$$
\left|w_{1}\right| \leq 1,\left|w_{2}-\mathrm{t} w_{1}^{2}\right| \leq 1+(|\mathrm{t}|-1)\left|w_{1}\right|^{2} \leq \max \{1,|\mathrm{t}|\},
$$

where $\mathrm{t} \in \mathbb{C}$. 


\section{$2 \quad$ Main result}

Our main result in this paper is stated as the following theorem.

Theorem 1 Let $\mathrm{f} \in \mathcal{A}$ of the form (1) belong to the class $\mathcal{F}(\mathcal{H}, \alpha, \delta, \mu)$. Then

$$
\left|\mathrm{a}_{2}\right| \leq \frac{2 \mathrm{t}}{\alpha \delta+(1-\alpha)(2-\mu)},
$$

and, for $\lambda \in \mathbb{C}$,

$$
\begin{aligned}
& \left|a_{3}-\lambda a_{2}^{2}\right| \leq \frac{t}{\alpha \delta+(1-\alpha)(3-2 \mu)} \max \left\{1, \mid 2 t\left(\frac{2 \lambda(\alpha \delta+(1-\alpha)(3-2 \mu))}{(\alpha \delta+(1-\alpha)(2-\mu))^{2}}\right.\right. \\
& \left.\left.-\frac{3+\frac{2(1-\alpha)(1-\mu)-\alpha\left(\delta^{2}-\mu^{2}\right)-\mu^{2}}{\alpha \delta+(1-\alpha)(2-\mu)}}{2(\alpha \delta+(1-\alpha)(2-\mu))}\right)-\frac{4 t^{2}-1}{2 t} \mid\right\} \text {. }
\end{aligned}
$$

Proof. Let $\mathrm{f} \in \mathcal{F}(\mathcal{H}, \alpha, \delta, \mu)$, then from (3) we have

$$
\alpha\left[\frac{z f^{\prime}(z)}{f(z)}\right]^{\delta}+(1-\alpha)\left[\frac{z f^{\prime}(z)}{f(z)}\right]^{\mu}\left[1+\frac{z f^{\prime \prime}(z)}{f^{\prime}(z)}\right]^{1-\mu}=\mathcal{H}(w(z), t), z \in \mathbb{U} .
$$

Using (2) and (4), we obtain

$$
\mathcal{H}(w(z), \mathrm{t})=1+\mathrm{U}_{1}(\mathrm{t}) w_{1} z+\left(\mathrm{U}_{2}(\mathrm{t}) w_{1}^{2}+\mathrm{U}_{1}(\mathrm{t}) w_{2}\right) z^{2}+\cdots .
$$

Making use of (1), (7) and (8), we get

$$
(\alpha \delta+(1-\alpha)(2-\mu)) a_{2}=U_{1}(t) w_{1},
$$

and

$$
\begin{aligned}
& 2(\alpha \delta+(1-\alpha)(3-2 \mu)) a_{3}+\frac{\alpha \delta(\delta-3)+(1-\alpha)\left(\mu^{2}+5 \mu-8\right)}{2} a_{2}^{2} \\
& \quad=U_{2}(t) w_{1}^{2}+U_{1}(t) w_{2} .
\end{aligned}
$$

From (9), we obtain

$$
\mathrm{a}_{2}=\frac{\mathrm{u}_{1}(\mathrm{t}) w_{1}}{\alpha \delta+(1-\alpha)(2-\mu)}
$$

Using Lemma 1 and (11), we obtain (5).

Putting (11) in (10), we have

$$
2(\alpha \delta+(1-\alpha)(3-2 \mu)) a_{3}=\mathrm{U}_{2}(\mathrm{t}) w_{1}^{2}+\mathrm{U}_{1}(\mathrm{t}) w_{2}
$$




$$
+\frac{\left(\alpha \delta(3-\delta)-(1-\alpha)\left(\mu^{2}+5 \mu-8\right)\right) \mathrm{u}_{1}^{2}(\mathrm{t}) w_{1}^{2}}{2(\alpha \delta+(1-\alpha)(2-\mu))^{2}} .
$$

Therefore,

$$
\begin{aligned}
a_{3} & =\frac{\mathrm{U}_{1}(\mathrm{t})}{2(\alpha \delta+(1-\alpha)(3-2 \mu))} \\
& \left(w_{2}+\left(\frac{\left(3+\frac{2(1-\alpha)(1-\mu)-\alpha\left(\delta^{2}-\mu^{2}\right)-\mu^{2}}{\alpha \delta+(1-\alpha)(2-\mu)}\right) \mathrm{U}_{1}(\mathrm{t})}{2(\alpha \delta+(1-\alpha)(2-\mu))}+\frac{\mathrm{U}_{2}(\mathrm{t})}{\mathrm{U}_{1}(\mathrm{t})}\right) w_{1}^{2}\right) .
\end{aligned}
$$

For $\lambda \in \mathbb{C}$, from (11) and (12) we obtain that

$$
\begin{gathered}
a_{3}-\lambda a_{2}^{2}=\frac{\mathrm{u}_{1}(\mathrm{t})}{2(\alpha \delta+(1-\alpha)(3-2 \mu))}\left(w_{2}+\frac{\mathrm{U}_{2}(\mathrm{t})}{\mathrm{u}_{1}(\mathrm{t})} w_{1}^{2}\right. \\
\left.-\mathrm{U}_{1}(\mathrm{t}) w_{1}^{2}\left(\frac{2 \lambda(\alpha \delta+(1-\alpha)(3-2 \mu))}{(\alpha \delta+(1-\alpha)(2-\mu))^{2}}-\frac{3+\frac{2(1-\alpha)(1-\mu)-\alpha\left(\delta^{2}-\mu^{2}\right)-\mu^{2}}{\alpha \delta+(1-\alpha)(2-\mu)}}{2(\alpha \delta+(1-\alpha)(2-\mu))}\right)\right) .
\end{gathered}
$$

Hence,

$$
\begin{aligned}
\left|a_{3}-\lambda a_{2}^{2}\right|= & \frac{t}{\alpha \delta+(1-\alpha)(3-2 \mu)} \mid w_{2}-\left(2 t \left(\frac{2 \lambda(\alpha \delta+(1-\alpha)(3-2 \mu))}{(\alpha \delta+(1-\alpha)(2-\mu))^{2}}\right.\right. \\
& \left.\left.-\frac{3+\frac{2(1-\alpha)(1-\mu)-\alpha\left(\delta^{2}-\mu^{2}\right)-\mu^{2}}{\alpha \delta+(1-\alpha)(2-\mu)}}{2(\alpha \delta+(1-\alpha)(2-\mu))}\right)-\frac{4 t^{2}-1}{2 t}\right) w_{1}^{2} \mid
\end{aligned}
$$

Using Lemma 1 for inequality (14), we obtain inequality (6).

Taking $\alpha=1-\beta, \delta=1$ and $\mu=0$ in Theorem 1 , we obtain the following result:

Corollary 2 [2] Let $\mathrm{f} \in \mathcal{A}$ of the form (1) satisfying the condition

$$
\left((1-\beta) \frac{z f^{\prime}(z)}{f(z)}+\beta\left(1+\frac{z f^{\prime \prime}(z)}{f^{\prime}(z)}\right)\right) \prec \mathcal{H}(z, t),
$$

where $\beta \in[0,1]$. Then

$$
\left|a_{2}\right| \leq \frac{2 t}{1+\beta},
$$


and, for $\lambda \in \mathbb{C}$,

$$
\left|a_{3}-\lambda a_{2}^{2}\right| \leq \frac{t}{1+2 \beta} \max \left\{1,\left|2 t\left(\frac{2 \lambda(1+2 \beta)}{(1+\beta)^{2}}-\frac{1+3 \beta}{(1+\beta)^{2}}\right)-\frac{4 t^{2}-1}{2 t}\right|\right\} .
$$

Taking $\alpha=0$ in Theorem 1 , we obtain the following result:

Corollary 3 [1] Let $\mathrm{f} \in \mathcal{A}$ of the form (1) satisfying the condition

$$
\left(\frac{z f^{\prime}(z)}{f(z)}\right)^{\mu}\left(1+\frac{z f^{\prime \prime}(z)}{f^{\prime}(z)}\right)^{1-\mu} \prec \mathcal{H}(z, t)
$$

where $\mu \in[0,1]$. Then

$$
\left|a_{2}\right| \leq \frac{2 t}{2-\mu},
$$

and, for $\lambda \in \mathbb{C}$,

$$
\left|a_{3}-\lambda a_{2}^{2}\right| \leq \frac{t}{3-2 \mu} \max \left\{1,\left|2 t\left(\frac{2 \lambda(3-2 \mu)}{(2-\mu)^{2}}+\frac{\mu^{2}+5 \mu-8}{2(2-\mu)^{2}}\right)-\frac{4 t^{2}-1}{2 t}\right|\right\} .
$$

\section{References}

[1] Ş. Altınkaya, S. Yalçın, On the Chebyshev coefficients for a general subclass of univalent functions, Turkish J. Math., 42 (2018), 2885-2890.

[2] Ş. Altınkaya, S. Yalçın, On the Chebyshev polynomial bounds for classes of univalent functions, Khayyam J. Math., 2 (1) (2016), 1-5.

[3] S. Bulut, N. Magesh, On the sharp bounds for a comprehensive class of analytic and univalent functions by means of Chebyshev polynomials, Khayyam J. Math., 2 (2) (2016), 194-200.

[4] J. Dziok, R. K. Raina, J. Sokol, Application of Chebyshev polynomials to classes of analytic functions, C. R. Acad. Sci. Paris, 353 (2015), 433-438.

[5] S. Kanas, O. Crişan, Differential subordinations involving arithmetic and geometric means, Appl. Math. Comput., 222 (2013), 123-131. 
[6] F. R. Keogh, E. P. Merkes, A coefficient inequality for certain classes of analytic functions, Proc. Amer. Math. Soc., 20 (1) (1969), 8-12.

[7] S. S. Miller, P. T. Mocanu, Differential Subordinations: Theory and Applications, Pure and Applied Mathematics, No. 225, Marcel Dekker, New York, 2000.

Received: May 21, 2019 